\title{
Anti Leishmanial Effect of Zinc Sulphate on the Viability of Leishmania tropica and L. major Promastigotes
}

\author{
Ali Fattahi Bafghi ${ }^{1, *}$; Mohammad Noorbala ${ }^{2}$; Mohammad Taghi Noorbala ${ }^{3}$; Mahdi \\ Aghabagheri $^{3}$ \\ ${ }_{1}^{1}$ Medical Parasitology and Mycology Department, School of Medicine, Shahid Sadoughi University of Medical Sciences, Yazd, IR Iran \\ ${ }^{2}$ School of Medicine, Shahid Sadoughi University of Medical Sciences, Yazd, IR Iran \\ ${ }^{3}$ Department of Dermatology, School of Medicine Shahid Sadoughi University of Medical Sciences, Yazd, IR Iran \\ ${ }^{*}$ Corresponding author: Ali Fattahi Bafghi, Department of Medical Parasitology and Mycology, School of Medicine, Shahid Sadoughi University of Medical Sciences, Shohadaye Gom- \\ nam Blv, Safaeeyeh, Postal Code: 8915173134, Yazd, IR Iran, Tel: +98-3518203410, Fax: +98-3518203414, E-mail: afbafghi@ssu.ac.ir \\ Received: July 2, 2013; Revised: September 21, 2013; Accepted: October 20, 2013
}

\begin{abstract}
Background: Cutaneous leishmaniasis (CL) is an endemic disease in developing countries. Although pentamidine orantimonite (Glucantime) has been recommended for cutaneous leishmaniasis treatment by the World Health Organization, there are some concerns too such as high cost, side effects, need for frequent injections, and restricted efficacy. Therefore, different methods have been used for CL treatment so far.

Objectives: This study assessed the sensitivity of two parasite agents of cutaneous leishmaniasis: Leishmania major and L. tropica to zinc sulphate in vitro. In the present study, the zinc sulphate effect on urban and rural strains of cutaneous leishmaniasis, viability of old world, in vitro is under investigation.

Materials and Methods: The design of the present study was experimental (laboratory-trial) based. Iranian endemic species of $L$. major and $L$. tropica were appropriately collected, proliferated, and maintained in the standard culture. Afterward, the proper concentrations of zinc sulphate were provided, sterilized, and added to the cultures containing parasites. In different intervals, parasites were counted by two methods: the slide and cell proliferation ELISA.

Results: Both parasite species showed sensitivity to zinc sulphate in vitro and in comparison with the control group, their numbers were reduced. Zinc sulphate (in concentrations of $0.5,1,2$, and 3 percent) was added to the cultures containing parasites, and the total number of the live parasites was counted through the slide method (Neubauer slide) every day up to the fifth day. The results were analyzed and found statistically significant $(\mathrm{P}<0.05)$. In the second phase, the counting process was repeated with the addition of zinc sulphate compound with different concentrations ( $3,4,5$, and 6 percent) and live parasite numbers were counted by ELISA method after 24 hours. The findings revealed that all the cultures containing zinc sulphate showed a slower growth in comparison to the control group. The mentioned difference was statistically significant $(\mathrm{P}<0.05)$.

Conclusions: Considering the safety of zinc sulphate compound in comparison with Glucantime, there is a possibility of using it in the treatment of CL caused by both species of L. major and L. tropica. It is obvious that more researches are mandatory both in vivo and in vitro to figure out its daily dosage, proper concentration, time and duration, and possible side effects.
\end{abstract}

Keywords:Cutaneous Leishmaniasis; Zinc Sulphate; In Vitro; Therapeutics

\section{Background}

Leishmaniasis is the name of a disease, which caused by several types of parasites. Each of the parasites is endemic to a specific geographical zone (1). The disease could be found in 88 different countries, and approximately 1.5 million of new cases are reported throughout the world annually; over $80 \%$ of the cases occur in the developing countries. Brazil, Iran, Afghanistan, and Sudan are the most infected countries. The disease itself is one of the priorities of hyperendemic areas. Morphologically, different species of parasite are identical and only differentiated by isoenzyme pattern and DNA analysis or monoclonal antibody (2). The clinical aspects are not enough to differentiate species. However, a special kind of disease or a distinct feature is possible to exist for some species.
Leishmaniasis parasite has an expanding cycle in the intestine of the female sandfly. During severe dermal infection in vertebrate host, amastigote form of the parasite exists in reticulated cells and leads to necrosis of mononuclear cells. The parasite shape is round and its length without flagellate is 2-3 $\mu \mathrm{m}$. In Romanowsky staining; cell kinetoplast gets deeply colorful and results in a particular view of organism. Leishmania species exists in sandfly body and cultures in the form of promastigote, which can move and has an anterior flagellate. Sandflies live in hot and wet areas, close to rodents' nests and trees holes. The infection is started with sandfly bite of exposed body areas typically during the night.

The parasite is zoophilic, and usually one type of it lives

Copyright (C) 2014,Ahvaz Jundishapur University of Medical Sciences; Published by Kowsar Corp. This is an open-access article distributed under the terms of the Creative Commons Attribution License, which permits unrestricted use, distribution, and reproduction in any medium, provided the original work is properly cited. 
in the body of some kind of a vertebrate animal, which is named as its reservoir host. Human is called as an accidental host, when becomes a reservoir host in facing with anthroponotic kind. Regarding the mentioned evidence, the geographical expansion of leishmaniasis occurs in special climates (3). Although the infection is in the forms of CL and visceral leishmaniasis (VL) in human, some VL infection can lead to skin complications too. In south and center of the America, Leishmania braziliensis parasite may cause skin complications, which result in mucosal or mucocutaneous leishmaniasis. Mucosal involvement in other Leishmania species is relatively rare (4).

Up to now, various treatments have been tried that none of them has had absolute results. Owing to the restrictive nature of the disease, the completion of cell immune system leads to the sore recovery in the final step. Incubation and clinical period are too variable, and takes from one to several months. The evaluation of the drugs is too difficult and needs scientific and standard methods, which are rarely used and most of the time, sore recovery is reported as a result of drug effect. Consequently, many believe that available drugs do not have a remarkable impact on disease treatment, and regarding the long course of the disease, there is not any absolute treatment.

In short, the treatment has gone three ways: 1) using physical treatments, including local surgery and removing necrotic tissues, cryotherapy, thermotherapy, X-ray therapy with different waves, and laser therapy; 2) using local drugs, including a long list of antimony compounds, antimalarial compounds e.g. emetine, corticosteroids, bleomycin, zinc sulphate, sodium chloride, and many other drugs solely or in combination are used with different results; 3 ) using systematic drugs, mainly the antimony compounds which their treatment results are very different from $100 \%$ effective to none. Other drugs, including antimalarial compounds, e.g. cumalar, different types of antibiotics specially macrolides, monomycine, neomycin, amphotericin, paromomycin, compounds like zinc in different compounds and potassium chloride sulfur compounds and Celina are reported more and less in disease treatment, which none of them has led to the complete recovery (4-6).

Anti leishmanial activities of zinc sulphate have been reported recently. The effect of zinc sulphate has been investigated many times in the forms of oral consumption or local injection into cutaneous lesions, which shows relatively positive but variable results. Therefore, the following steps must be carried out and investigated at first: the effect of the zinc sulphate compound on different species of parasite in vitro (with different dosages and different methods and intervals), and then in vivo and finally on human. And in later steps, there might be some hope to find a more effective treatment for the disease. We would like to mention that newer immunotherapy methods and probes are underway to prepare the vaccine for this disease, but acceptable results have not been reported yet. In this regard, to assess the relationship between CL and different gradients of zinc sulphate, we cultured Leishmania species and studied antimicrobial properties of zinc sulphate compound in vitro (7-18).

\section{Objectives}

This study assessed the sensitivity of two parasite agents of CL; L. major and L. tropica to zinc sulphate compound in vitro. In other words, the aim of the study was to evaluate the efficacy of zinc sulphate compound on urban and rural strains of CL (viability of old world) in vitro.

\section{Materials and Methods}

The present study is experimental (laboratory-trail) and conducted in several steps.

\subsection{The Preparation of Parasite}

L.major strain [MRHO/IR/75/ER] promastigote (PM) stage was collected from the Medical Parasitology Department, School of Medical Sciences, Tehran Tarbiat Modares University. Then, it was proliferated in Novy-Nicolle-Mac Neal (NNN) (Iran) and RPMI1640 (Gibco, SinaQuia Gen, USA) media with high density according to the instruction of Limoncu et al. (19).

L. major strain (MRHO/IR/75/ER) and L. tropica were maintained in BALB/c mice. Amastigotes were isolated from mice spleens, and transformed to PM in NNN medium. Subsequently, with the third PM passage from NNN medium, they were progressively adopted to RPMI 1640 media, which increasingly supplemented with antibiotics, glutamine and fetal calf serum (FCS). The complete medium consisted of penicillin $(100 \mathrm{U} / \mathrm{mL})$, streptomycin $(100 \mu \mathrm{g} / \mathrm{mL})$, and $20 \%$ heat-inactivated fetal calf serum (Gibco, SinaQuia Gen, USA) at $25^{\circ} \mathrm{C}(20)$.

\subsection{Zinc Sulphate Preparation}

Zinc sulphate heptahydrate, $\mathrm{ZnSO} 4.7 \mathrm{H}_{2} \mathrm{O}$ (BDH, Iran) was dissolved in distilled water in known concentration and autoclaved at $121^{\circ} \mathrm{C}$ for 20 minutes before use. Ampules of melamine antimonite (Glucantime) purchased from Spica, France.

\subsection{Parasite Counting}

\subsubsection{The Slide Method}

A fixed initial density of the parasites was transferred to screw-capped vials containing 5 mL of RPMI 1640 with different concentrations of zinc sulphate compound $(0.5 \%$, $1 \%, 2 \%$ and $3 \%$ ), then melamine antimonite added and for each concentration, three vials were chosen, as well as a control vial for each run. The vials were then incubated at $26^{\circ} \mathrm{C}$ for PM. On the next four days, the cultures were counted. A 1:10 diluted saline solution along with the appropriate dye was prepared. The PMs permeable to the blue dye got died while viable ones exclude the dye (11). The chamber of a Neubauer slide was charged and the 
Fattahi Bafghi A et al.

number of organisms in 16 small corner squares was counted. The total number per $\mathrm{mL}$ is equal to $\mathrm{N}$ (counted) times10 (number in $1 \mathrm{~mm} 3 \times 103$ number $1 \mathrm{~mL}$ ) times10 (dilution factor).

The $\mathrm{LD}_{50}$ (Lethal dose 50\%) was calculated according to the method of Healy et al. (12). This method was first described by Sharquie et al. (13). A modified method was used in the present study, and the obtained results were compared with gained ones by the previous method of $L$. major. The appropriate dye was added to a dilution of the parasites growing on liquid medium. Trypan blue $0.4 \%$ was used for PM. One drop containing the parasites was put on a slide along with a drop of a drug solution and covered by a cover slip. The slides were examined under the light microscope, and the percentage of stained parasites was noted. Normal saline was used as the control (11, $12,21,22)$.

\subsubsection{The Cell Proliferation ELISA, BrdU Method}

The cell proliferation ELISA, and BrdU (Chemiluminescent) was performed as instructed by Roche Diagnostics GmbH Roche Applied Science 68298 Mannheim Germany (Version March 2005, Cat. No. 11669915 001) which is briefly as follows: 1) a fixed initial density of the parasites was transferred to screw-capped vials containing $5 \mathrm{~mL}$ a liquid medium with different concentrations of zinc sulphate $(0.5,1,2,3,4,5$, and 6 percent $)$ and melamine antimonite and each density was done in triplicates. Each run also had control vials; 2 ) the cell proliferation method of ELISA, and BrdU was conducted according to the Kit instruction (13). Finally, all data were collected to be analyzed through statistical procedures.

\subsection{Data Analyses}

Data are expressed as Mean \pm SD of lesion's diameter and animal's weight, and were statistically analyzed by multivariate tests for evaluating the effect of time within-subjects and multiple comparisons test for comparing the variables between groups. In this study, $\mathrm{P}<0.05$ was considered as the significant level.

\section{Results}

$\mathrm{LD}_{50}$ of studied ZS and Glucantime on $L$. major and $L$. tropica PMs are as follows: the LD50 for zinc sulphate was $221.9 \mathrm{mg} / \mathrm{mL}$ or $44.4 \mathrm{mg} / \mathrm{Ml}$ for $\mathrm{Zn}^{++}$, and the $\mathrm{LD}_{50}$ for pentavalent antimony compounds is $334.7 \mathrm{mg} / \mathrm{mL}$ (Table 1 ).

Table 1. The $\mathrm{LD}_{50}$ of Zinc and Pentavalent Antimony Against $L$. major and L. tropica PMs

\begin{tabular}{lcc}
\hline Organism & $\mathbf{Z n}^{++}, \mathbf{m g} / \mathbf{m L}$ & Glucantime, $\mathbf{m g} / \mathbf{m L}$ \\
\hline Leishmania major PM & 44.4 & 334.7 \\
Leishmania tropica PM & 32.4 & 2250 \\
\hline
\end{tabular}

The LD50 Zine and Pentavalent Antimony against L(L)major and L(L)tropica PMs

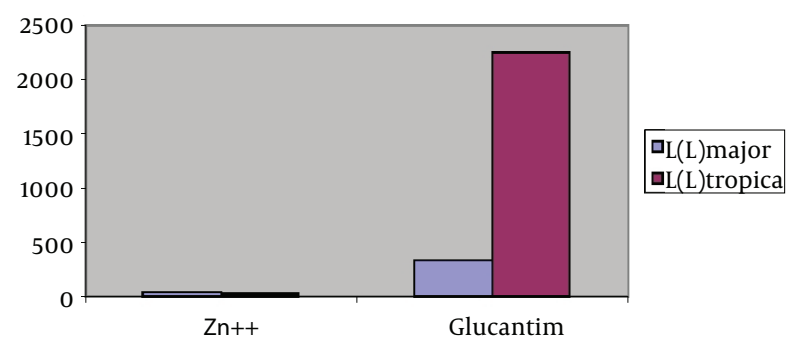

Figure 1. The $\mathrm{LD}_{50}$ of Zinc and Glucantime Against L. major and L. tropica PMs

The zinc sulphate effect on urban and rural strains of CL (Viability of old world) in vitro was investigated and the parasites were counted in different time intervals, by two methods of the slide and cell proliferation ELISA, BrdU. Figure 1 shows the effect of different concentrations of zinc sulphate against L. major PMs. Zinc, in geometrically increasing concentration, inhibited the growth of L. major PMs.

\subsection{The Zinc Sulphate Effect on Rural Strains L. ma- jor and L. tropica in Vitro by the Slide Method}

The findings of zinc sulphate effect on rural strains Leishmania. L. major with densities of $0.5,1,2,3$ on the first, second, third, fourth, and fifth day of application showed that the zinc sulphate increase has an inverse relation with the parasite proliferation percentage. In other words, while the parasites in the control group showed a typical growth procedure, the parasites in the experimental groups revealed a much slower growth rate. Interestingly, the growth percentage is different in different zinc sulphate concentrations and with its increase, the parasite proliferation decreased remarkably. In other words, the growth and proliferation of the parasites were strictly controlled by the drug (Table 2 and Figure 2).

The mentioned findings were analyzed by repeated measures analysis of variance (ANOVA), and the final output showed that the zinc sulphate compound has a statistically significant effect on the growth of L. major ( $\mathrm{P}<$ 0.001).

Table 2. Mean Frequency of Viability of PM of L. major [MRHO/ IR/75/ER] in Culture According to Zinc Sulphate Gradients in Due Times Comparing With Control Group by the Slide

\begin{tabular}{lllll}
\hline Densities, d Control & $\mathbf{0 . 5} \%$ & $\mathbf{1} \%$ & $\mathbf{2} \%$ & $\mathbf{3} \%$ \\
\hline
\end{tabular}

\begin{tabular}{lccccc}
\hline 1st & 78 & $61 \pm 2.82$ & $46 \pm 2.82$ & $44 \pm 1.41$ & $20 \pm 0.70$ \\
2nd & 247 & $76 \pm 2.12$ & $64 \pm 1.41$ & $54 \pm 2.82$ & $33 \pm 0.707$ \\
3rd & 294 & $94 \pm 1.41$ & $91 \pm 5.65$ & $62 \pm 1.41$ & $41 \pm 0.707$ \\
\hline 4th & 387 & $101 \pm 2.82$ & $89 \pm 5.65$ & $68 \pm 1.41$ & $48 \pm 1.41$ \\
\hline
\end{tabular}




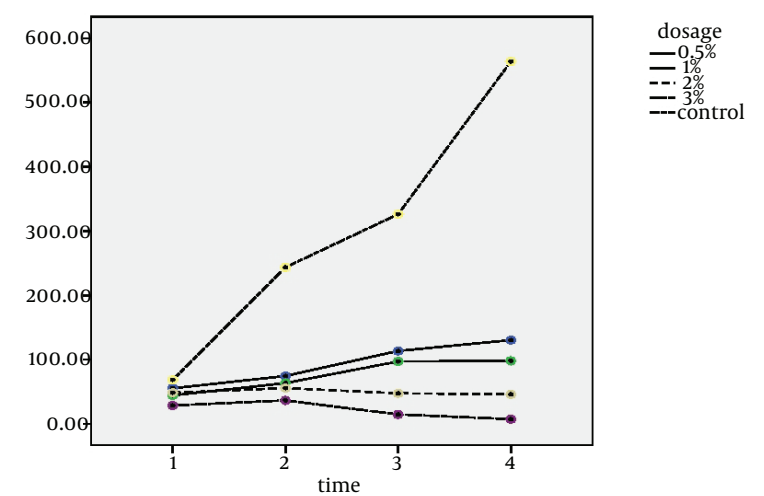

Convariates appearing in the model are evauated at the following values:time $0=36.6667$

Figure 2. Mean Frequency of Viability of PM of L. major [MRHO/IR/75/ER] in culture According to Zinc Sulphate Concentrations in Due Times Comparing With the Control Group by the Slide

To investigate the effect of different concentrations of zinc sulphate compound on L. major viability, four groups with different densities without the control group consideration were statistically analyzed by repeated measures ANOVA and $(\mathrm{P}=0.001)$ showed that the effect was significant. It means that the drug increase (higher concentration) has led to a significant parasite decrease (growth inhibition).

\subsection{The Zinc Sulphate Effect on Rural Strains. L. ma- jor and L. tropica in Vitro by ELISA, BrdU Method}

Considering the results obtained from the slide method of parasite proliferation and given the fact that increase in zinc sulphate compound inhibits the parasites' growth, the drug concentration in ELISA, BrdU was up to $6 \%$. The findings showed that in the culture of L. major with zinc sulphate compound, the growth and proliferation were strictly affected and dropped. In other words, the parasitic growth decrease has a direct relationship with zinc sulphate concentration, and with the increase of zinc sulphate, there is an inhibition in parasite growth. Interestingly, in the concentration of $6 \%$, the highest inhibition level was observed.

To put it another way, considering the time intervals, parasite population in the control group have been remarkably increased. By contrast, in the groups containing the zinc sulphate compound, the parasite growth was decreased. The most remarkable inhibition was seen in the last group containing $6 \%$ of zinc sulphate compound. It means that an increase in zinc sulphate compound was strictly inhibited the parasite growth in vitro (Table 3 and Figure 3). The growth and proliferation of L. major in experimental groups containing the zinc sulphate in comparison with the control group showed less increase.
It was tested by repeated measures ANOVA, and the result was statistically significant $(P=0.001)$. In addition, the results of different groups of zinc sulphate compounds were compared with each other, and the result was statistically significant $(\mathrm{P}=0.001)$.

Table 3. Mean Frequency of Viability of PMs of L. tropica in Culture According to Zinc Sulphate Concentration in Due Times Comparing With Control Group by the Slide

\begin{tabular}{lrrrrr}
\hline Concentration, d & Control & $\mathbf{0 . 5} \%$ & $\mathbf{1} \%$ & $\mathbf{2} \%$ & $\mathbf{3} \%$ \\
\hline $\mathbf{1}^{\text {st }}$ & 94 & $92 \pm 2.12$ & $84 \pm 0.707$ & $68 \pm 1.41$ & $58 \pm 1.41$ \\
$\mathbf{2}^{\text {nd }}$ & 127 & $105 \pm 0.707$ & $98 \pm 1.41$ & $92 \pm 2.12$ & $85 \pm 1.41$ \\
\hline 3rd & 188 & $107 \pm 1.41$ & $114 \pm 1.41$ & $100 \pm 1.41$ & $86 \pm 1.41$ \\
$\mathbf{4}^{\text {th }}$ & 272 & $126 \pm 1.41$ & $132 \pm 3.53$ & $123 \pm 2.12$ & $94 \pm 1.41$ \\
\hline
\end{tabular}

The comparison of optical density mean of parasite $L$. major the in the culture with different concentrations was conducted by Kruskal-Wallis Test which was statistically significant among different concentrations and control group $(\mathrm{P}=0.001)$. The ELISA, BrdU findings with different concentrations of zinc sulphate compound without considering the control group was investigated. The comparison of optical density mean in the case of parasite (L.tropica) in the culture with different densities was conducted by Kruskal-Wallis test leishmania which it was a statistically different significant among concentrations $(\mathrm{P}=0.02)$. It means with the increase in concentration; the inhibition effect on the parasite increases too.

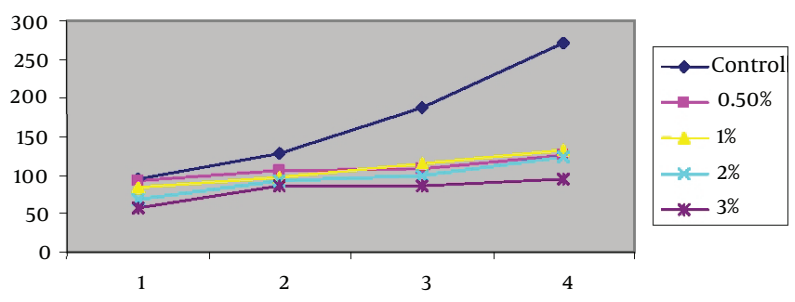

Figure 3. Mean Frequency of Viability of PM of L. tropica in Culture According to zinc sulphate concentrations in Due Times Comparing With Control Group by the Slide.

Table 4. The Effect of Zinc Sulphate on Rural \& Urban Strains of Cutaneous Leishmaniasis, Viability of Old World in Vitro

\begin{tabular}{lccccc}
\hline $\begin{array}{l}\text { Species, } \\
\text { Concentration }\end{array}$ & Control & $\mathbf{0 . 5}$ & $\mathbf{1}$ & $\mathbf{2}$ & $\mathbf{3}$ \\
\hline L.major & 0.16733 & 0.14067 & 0.12367 & 0.11733 & 0.092 \\
L.tropica & 0.14900 & 0.12467 & 0.11433 & 0.10733 & 0.09167 \\
\hline
\end{tabular}




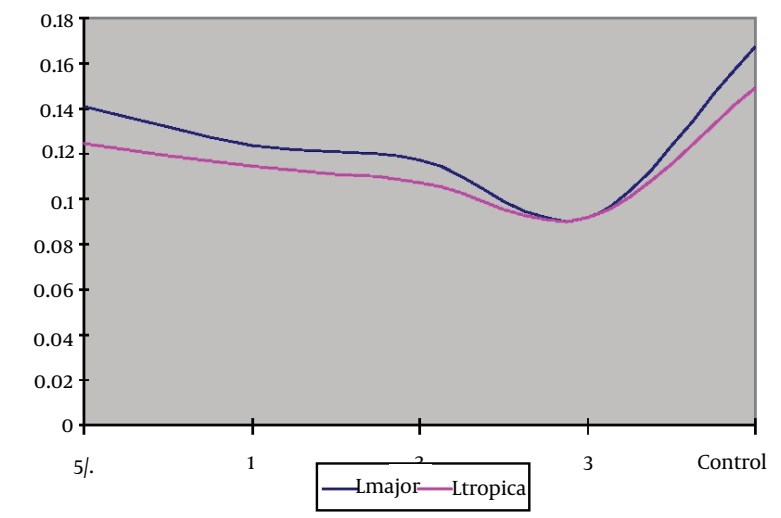

Figure 4. The effect of Zinc Sulphate on Rural \& Urban Strains of Cutaneous Leishmaniasis, Viability of Old World in Vitro.

Table 5. Staining Mean of Urban and Rural PM in Culture on Cell Proliferation, ELISA Method ${ }^{\mathrm{a}}$

\begin{tabular}{lcc}
\hline Species & L. major & L. tropica \\
\hline $\begin{array}{l}\text { Zinc Sulphate Concen- } \\
\text { trations, \% }\end{array}$ & & \\
\hline Control & $0.167 \pm 0.005$ & $0.149 \pm 0.003$ \\
\hline 0.5 & $0.140 \pm 0.003$ & $0.124 \pm 0.003$ \\
\hline 1 & $0.123 \pm 0.006$ & $0.114 \pm 0.002$ \\
\hline 2 & $0.117 \pm 0.004$ & $0.107 \pm 0.004$ \\
\hline 3 & $0.092 \pm 0.006$ & $0.091 \pm 0.003$ \\
\hline
\end{tabular}

${ }^{\mathrm{a}}$ Data are presented as mean $\pm \mathrm{SD}$

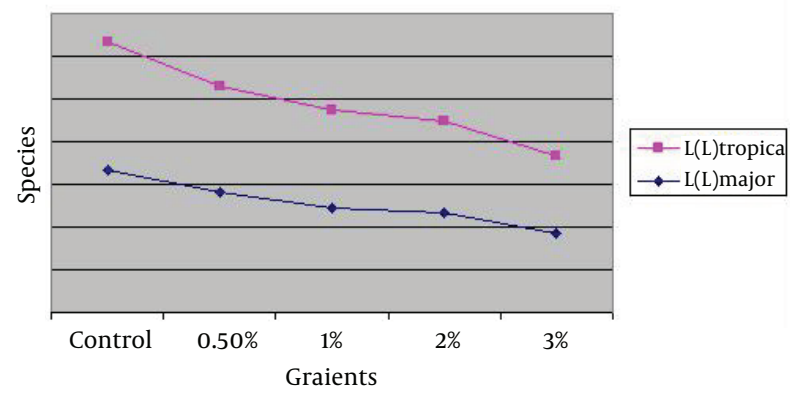

Figure 5. Staining Mean of Urban \& Rural PM in Culture on Cell Proliferation, ELISA Method

\section{Discussion}

The findings of the present study revealed that zinc sulphate compound had a protective effect on the promastigote form of the parasites (two strains of urban and rural leishmaniasis), and stopped their proliferation in vitro. Different methods to cure leishmaniasis have been recommended so far that most of them comprised topical and systemic treatments. The four- and five-valentantimony compounds have been introduced since 1940 for systemic treatment and the five capacity compounds have been used as the first drug of choice in the treatment since 1984 . The mentioned drug is relatively expensive and has some severe side effects. Besides, there are many reported cases of its inefficacy in wound recovery. Owing to the reasons, various drugs have been used so far, including sodium chloride and zinc sulphate.

The first report of efficacy of $2 \%$ zinc sulphate compound dates back to a study in 2010) (17) in which zinc sulphate compound with a concentration of 100 to 400 $\mathrm{mL} / \mathrm{kg}$ was given to the mice. The results showed that the experimental group in comparison with the control one suffered less and the disease course was less severe too (18). The anti leishmanial effect of zinc sulphate is not completely clear. It might be the effect of zinc sulphate on different enzymes, which interfere in DNA and proteins synthesis. Furthermore, it was reported that zinc can deactivate DNA enzyme of herpes virus Thus, it is more probable that the zinc main effect is on enzymes, which play some role in nucleic acids metabolism. As a result, zinc sulphate compound stops the parasite proliferation in vitro. The present study showed the direct effect of drug on parasite. However, more precise studies for revealing the exact effect mechanism of the drug are mandatory.

Zamani Sorkhroodi et al. conducted a study in 2010 (23). Thirty-three mice were infected by L. major, and then they were divided into three groups by random. The first group was received $0.35 \mathrm{~mL} / \mathrm{kg}$ solenoid sodium for 30 days; second group received $2 \mathrm{mg} / \mathrm{kg}$ zinc sulphate compound for 30 days; and the third group received $10 \mathrm{~mL} /$ $\mathrm{kg}$ distilled water (control group). All the groups received $60 \mathrm{mg} / \mathrm{kg}$ standard Glucantime injection for 14 days. The size of the wound in solenoid sodium receiving group got bigger, and in the second group it stopped enlarging but no significant difference between zinc sulphate group and distilled water group was observed. The findings of the study showed that zinc sulphate compound has no significant effect on disease control in laboratory animals (19). Regarding these results, the reduplication of experiments of drug effects in vitro was needed.

Many researchers have focused on the drug effects on human beings. In 1998, Rafid et al. (24) used zinc sulphate compound through injection in animate wound and reported the positive results. Sixty-three patients suffered from CL were divided into four groups by random. Three groups received $2 \%$ zinc sulphate compound, $7 \%$ colored sodium, and antimony injection into the wound, respectively. Fourth group was the control one which received no treatment. At the end of 45-day treatment, the results revealed that the first three groups obtained comparable treatment outputs and zinc sulphate compound group with $94.8 \%$ recovery showed the best result (20). Another study was conducted by Sharquie et al. (25) in 
2001. They put 104 patients diagnosed with $L$. major into four groups. Three groups received oral dosages of 2.5 , 5 , and $10 \mathrm{~mL} / \mathrm{kg}$ zinc sulphate compound, and fourth group, which was the control one did not receive any treatment. All the patients were observed for 45 days, and finally, the results showed that clinical and laboratory recovery in the groups of 2.5, 5, and 10 was 83.9, 93.1, and 96.9, respectively. None of the patients in the control group showed any cure.

The obtained result emphasized on the efficacy of oral zinc sulphate compound as an appropriate and harmless medication in treatment of CL (11). The findings of mentioned studies which were conducted in Iraq showed the positive effect of the drug, let alone the studies of drug effect on the parasite in vitro, and animal models which were conducted in this country. However, many studies that were conducted in Iran did not report the drug efficacy. In 2007, a study was conducted by Yazdanpanah et al. (26) in Mashhad to observe the efficacy of oral zinc sulphate compound on cutaneous leishmaniasis. Thirtyone patients were treated by taking $10 \mathrm{mg} / \mathrm{kg}$ oral zinc sulphate compound during 45 days. Twenty-two patients completed the treatment and only two of them showed perfect recovery. Therapeutic value of oral zinc sulphate in cutaneous leishmaniasis treatment was assessed as insignificance by conductors (12). Another study was conducted by Iraji et al. (27) in Isfahan in 2004. In this study 104 patients suffered from severe CL participated and were divided into two groups by random. One group received $2 \%$ zinc sulphate compound, and the other group received antimony for a total of six weeks. A total of 66 patients, 35 persons form antimony group and 31 persons from zinc sulphate completed the treatment. The recovery percentage in the antimony group was $60 \%$ and in the zinc sulphate group was $83.8 \%$. In the end, the conductors came to this conclusion that the injection of $2 \%$ zinc sulphate into the wound can be the alternative treatment for CL.

Another study was conducted by Firooz et al. (28) in 2005. A total of 72 patients suffered from severe urban CL were selected and divided into two groups. One group received zinc sulphate compound and the other one received Glucantime injection weekly. 35 patients (13 persons from zinc sulphate group and 22 ones from Glucantime) completed the treatment. The complete recovery was observed one week after the treatment completion in 2 and 19 patients in zinc sulphate group and Glucantime group, respectively. The findings revealed that the zinc sulphate injection in comparison with Glucantime had fewer side effects (22).

Various studies emphasized on the mechanism of zinc sulphate action on laboratory animals and human being regarding the change of immune response. The shortage of this component changes the immune response of a cell from Th1 toward Th2 i.e. humoral immune response. Considering the fact that the Th1 immune response leads to the production of items such as IL2 and INF $\gamma$, which control virus infections and other inside-cell pathogens and as a result is more effective in comparison with Th2 response (13). Also, the zinc sulphate role in determining molecular construction of Leishmania is important, which affects joining and entering the Leishmania into the cells. In several studies, low levels of zinc serum, selenium and iron accompanying with a decrease in related enzymes activity such as flotation peroxides and catalase were reported $(29,30)$. Two other studies focusing on the dogs suffered from VL, and human beings suffered from leishmaniasis showed the low level of zinc and iron and other cytotoxic compounds related to these elements (30).

\section{Acknowledgements}

The authors thank all people who have assisted the ex $\neg$ perimental procedure, especially Mrs. Mahin Ghafourza $\neg$ deh, Msc in medical Parasitology and Marziyeh Beigom.

\section{Authors' Contributions}

Ali Fattahi Bafghi: selected and presented the basic theme of the article, literature review and writing of the manuscript, Mohammad Noorbala: data collection, Mohammad Taghi Noorbala: supervised the study and Mahdi Aghabagheri: edition of manuscript.

\section{Funding/Support}

This study was supported by the grant of Yazd Shahid Sadoughi University of Medical Sciences.

\section{References}

1. Ghosh MK, Dériaud E, Saron MF, Lo-Man R, Henry T, Jiao X, et al. Induction of protective antiviral cytotoxic $\mathrm{T}$ cells by a tubular structure capable of carrying large foreign sequences. Vaccine. 2002;20(9-10):1369-77.

2. Vega-López F, Hay RJ. Parasitic Worms and Protozoa. In: Burns T, Breathnach S, Cox N, Griffiths C editors. Rook's Textbook of Dermatology. 8th ed. united State: Wiley-Blackwell;2010. pp. 33-9.

3. Bryceson ADM. Clinical variations associated with various taxa of Leishmania.Montpelier: CollInt CNRS/INSERM; 1984.

4. Warrell DA, Cox TM, Firth JD. Oxford Textbook of Medicine. oxford: Oxford University Press; 2003.

5. Rioux JA, Groubert JR, Lanotte G. A case of leishmanioseautochtone lamuqueusenasale. In the Ent Papers. 1980;15:423-5.

6. Bryceson A. Therapy in man. In: Peters W, Killick-Kendrick R editors. The Leishmaniases in Biology and Medicine. London: Academic Press; 1987. pp. 56-61.

7. Neva FA, Petersen EA, Corsey R, Bogaert H, Martinez D. Observations on local heat treatment for cutaneous leishmaniasis. Am J Trop Med Hyg. 1984;33(5):800-4.

8. Bassiouny A, El Meshad M, Talaat M, Kutty K, Metawaa B. Cryosurgery in cutaneous leishmaniasis. BrJDermatol.1982;107(4):467-74.

9. Currie MA. Treatment of cutaneous leishmaniasis by curettage. BMJ. 1983;287(6399):1105-6.

10. Sanchez-Moreno M, Gomez-Contreras F, Navarro P, Marin C, Ramirez-Macias I, Olmo F, et al. In vitro leishmanicidal activity of imidazole- or pyrazole-based benzo[g]phthalazine derivatives against Leishmania infantum and Leishmania braziliensis species. J Antimicrob Chemother. 2012;67(2):387-97.

11. Jaffe CL, Grimaldi G, McMahon-Pratt D. The cultivation and cloning of Leishmania. In: Morel CM editor. Genes and antigens of parasites: a laboratory manual : the proceedings of a course held at the Oswaldo Cruz Institute, FIOCRUZ, Rio de Janeiro, Brazil.. Oswaldo 
Cruz, Rio de Janeiro: Instituto Oswaldo Cruz, Fundação Oswaldo Cruz; 1987. pp. 47-90.

12. Healy MJR. Estimating LD50s without a computer. Parasitol Today. 1988;4(1):25-7.

13. Sharquie KE. A new intralesional therapy of cutaneous leishmaniasis with hypertonic sodium chloride solution. J Dermatol. 1995;22(10):732-7.

14. Roche Applied Science . Cell Proliferation ELISA, BrdU (chemiluminescent) For life science research only. Not for use in diagnostic procedures for in vitro use only: Roche Applied Science; 2005.

15. Rodriguez-Cortes A, Ojeda A, Francino O, Lopez-Fuertes L, Timon $\mathrm{M}$, Alberola J. Leishmania infection: laboratory diagnosing in the absence of a "gold standard". Am J Trop Med Hyg. 2010; 82(2):251-6.

16. Sharquie KE, Hameed AF. Panniculitis Is an Important Feature of Cutaneous Leishmaniasis Pathology. Case Report Dermatol Med. 2012;2012:1-4.

17. Sorkhroodi FZ, Naeini AA, Ramazani AZ, Ghazvini MA, Mohebali M, Keshavarz S. Therapeutic effect of sodium selenite and zinc sulphate as supplementary with meglumine antimoniate ( glucantime(R)) against cutaneous leishmaniasis in BALB/c mice. Iran J Parasitol. 2010;5(3):11-9.

18. Iraji F, Vali A, Asilian A, Shahtalebi MA, Momeni AZ. Comparison of intralesionally injected zinc sulfate with meglumine antimoniate in the treatment of acute cutaneous leishmaniasis. Dermatology. 2004;209(1):46-9.

19. Limoncu ME, Ozbilgin A, Balcioglu IC, Ozbel Y. Evaluation of three new culture media for the cultivation and isolation of Leishmania parasites. J Basic Microbiol. 2004;44(3):197-202.

20. Ihalamulla RL, Rajapaksa US, Karunaweera ND. Microculture for the isolation ofLeishmaniaparasites from cutaneous lesions - Sri Lankan experience. Annals Trop Med Parasitol. 2005; 99(6):571-5.
21. Sharquie KE. A new Hypotheses. J Dermatol. 1995;49:1-14.

22. Puentes F, Guzman F, Marin V, Alonso C, Patarroyo ME, Moreno A. Leishmania: fine mapping of the Leishmanolysin molecule's conserved core domains involved in binding and internalization. Exp Parasitol.1999;93(1):7-22.

23. Zamani Sorkhroodi F, Alavi Naeini AM, Zahraei Ramazani AR, Aghaye Ghazvini MR, Mohe bali M, Keshavarz SA. Therapeutic Effect of Sodium Selenite and Zinc Sulphate as Sup- tary with Meglumine Antimoniate ( Glucantime $囚$ ) Against Cutaneous Leishmaniasis In BALB/C Mice. Iran J Parasitol. 2010;5(3):11-9.

24. Rafid AN, Khalifa ES, Imad BF. Zinc Sulphate in the Treatment of Cutaneous Leishmaniasis: an in Vitro and Animal Study. Mem Inst Oswaldo Cruz, Rio de Janeiro. 1998;93(6):831-7.

25. Sharquie KE, Najim RA, Al-Hayani RK, Al-Nuaimy AA, Maroof DM The therapeutic and prophylactic role of oral zinc sulfate in management of recurrent aphthous stomatitis (ras) in comparison with dapsone. Saudi Med J. 2008;29(5):734-8.

26. Yazdanpanah $\mathrm{K}$. The effect of backgro und knowledge and reading comprehension test items on male and female performane. The Reading Matrix. 2007;7(2).

27. Iraji F, Vali A, Asilian A, Shahtalebi MA, Momeni AZ. Dermatology. Dermatology. 2004;209(1):46-9.

28. Firooz A, Khatami A, Khamesipour A, Nassiri-Kashani M, Behnia $\mathrm{F}$, Nilforoushzadeh $\mathrm{M}$, et al. Intralesional injection of $2 \%$ zinc sulfate solution in the treatment of acute old world cutaneous leishmaniasis: a randomized, double-blind, controlled clinical trial.J Drugs Dermatol. 2005;4(1):73-9.

29. Sprietsma JE. Zinc-controlled Th1/Th2 switch significantly determines development of diseases. Med Hypotheses. 1997;49(1):1-14.

30. Kocyigit A, Erel O, Seyrek A, Gurel MS, Aktepe N, Avci S, et al. Effects of antimonial therapy on serum zinc, copper and iron concentrations in patients with cutaneous Leishmaniasis in Turkey. J Egypt Soc Parasitol. 1998;28(1):133-42. 